\title{
Packaging Design with Hidden Near Infrared Colour Separation
}

\author{
Jana ŽlLJAK, Denis JUREČIĆ, Vilko ŽILJAK
}

\begin{abstract}
We present extended visual and infrared colour management with the application in packaging print in this paper. Visual design is a vector graph which is masked by pixel graph according to a plan which altogether allows visibility of packaged product. The hidden information, invisible to the naked eye, is printed on the transparent foil and is identified by an infrared camera. Two independent pieces of pictural information are made with the "dualcolours". Each tone of colour consists of two different process components which respond differently in near infrared spectrum. Duality of graphic design for visual (V) and near infrared (Z) spectrum is designed for the print with process colours. The recipes for dual colorants are achieved by near infrared spectroscopy. A new mathematical regression model is provided for transparent material which is applied in food packaging. The infrared vector graphics increases safety quality of packaging technology; against copying, scanning and subsequent reproduction.
\end{abstract}

Keywords: dualcolours; food packaging; hidden image; near infrared spectroscopy; VZ design

\section{INTRODUCTION}

The special GCR (grey component replacement) method is introduced and is called VZ separation. That dye separation is subordinated to the idea of joining two images [1]. The first image is intended for the visual spectrum while the second image will be identified by a near infrared (ZRGB) camera [2]. The second image is a mask carrying information where $V$ component of colorant is joining the vector line and where this line is continuing to be coloured by $\mathrm{Z}$ colour. We introduce separation with the control of "empty space" between lines in the paper. The space between the lines remains uncoloured in order to increase the visibility of packed product.

The colour in the vector graphics is observed in two conditions of colour components for the same colour tone. In VZ separation method, the $K$ component (carbon black) has been given before execution of the GCR method with the fixed value of $30 \%$ coverage of $K$ colour. Double infrared information introduces variables "V" (visual RGB spectrum) and $Z$ as a measure of absorption of the NIR light at $1000 \mathrm{~nm}$ [2].

We present the new VZ mathematical model for the colouring material for packaging as duality of graphics for the visual and NIR spectrum in this paper. Each material and associated colours look for their own algorithm for VZ separation. The first models of INFRAREDESIGN ${ }^{\circledR}$ (IRD) procedure of double image are developed for the area of documented products printed on paper. The graphics in the visual and infrared spectrum on documents are observed in only one colour without hiding the $\mathrm{Z}$ image [3]. The hidden camouflage design on the textile has its own specificity due to the extreme colour penetration into the material [4]. Multicolour solutions with image separation in individual blockages of sun spectrum are observed in the paper. VZ transparent vector graphics has been improved with the display of a dual image as a line drawing that is masked by pixel graphic.

\section{VZ COLOUR AREA WITH THE EXTENSION TO THE NEAR INFRARED SPECTRUM}

We are defining colours in two groups as $V$ and as $Z$ through their qualities of light absorption in the range of
400 to $1000 \mathrm{~nm}$. We differentiate two colorant conditions, two different components for the same colour tone [5].

The different colour tones are set as $V$, and they are created from the dyes that do not absorb near infrared light. Dyes with the positive absorption value of $Z$ (on $1000 \mathrm{~nm}$ ) are called $Z$ dyes. A set of two dyes that have an equal value in $\mathrm{V}$ spectrum (same spectrograms in the RGB colour space), but different $Z$ values (in the near infrared), are called "VZ dual colours". We are introducing "dual colouring" with VZ characteristics. The VZ separation has opened a new graphical area with the title: "Hidden images". VZ separation is a new way of determining the significance of $K$ component in GCR procedure. Therefore, a pure $K$ channel is the default graphics for recognizing by infrared camera. The dual VZ colours have two separate values: positive NIR absorption carbon black dyes in $Z$ colorant, or zero percent of coverage $K$ for $V$ colours. Both colours provide an equal RGB colour experience in the visual spectrum. $30 \%$ coverage of $K$ channel is enough to distinguish between the $V$ graphic from one-tone $Z$ graphic.

Experiments have been carried out in the iterative experimental design in order to achieve a satisfactory formulation of dualcolours. $\Delta E$ is only a single numerical criterion of colour equality, but only in a $V$ range. The spectrogram shows light absorption continuity from 400 to $900 \mathrm{~nm}$. Spectrogram indicates the direction for fixing the components of colorant in order to achieve VZ criterion of dual dyes.

The technology of dual colours introduces a specific security image in the $\mathrm{K}$ channel for process print. This procedure allows the existence of two images at the same place. The control of sunlight absorption of these spectra has been introduced since the $Z$ dyes respond also outside the range of the human eye (in the near infrared spectrum). An additional hidden image is viewed by an infrared camera for the VZ colour separation. The image is target planned, designed for near infrared spectrumwhile it is invisible in the $V$ (visual) spectrum. The $V$ and $Z$ dualcolours, named also the "IRD twins", as a method, is the procedure for replacing CMY with a colour which in its own way benefits from the diversity of light absorption qualities of process colours.

Pairs of twin dyes form the colour space in order to determine the mathematical model as an analytical term in the dependency of $Z$ dyes on $V$ dyes. In all experiments, 
carbon black has a constant coverage by $30 \%$ and is located in the $\mathrm{Z}$ colorant. The other $Z_{30}$ components were experimentally determined. Eighty experiments were accepted and the results were analysed with the regression procedure. A system of equations dependency $Z_{30}$ on default $V$ dyes is provided here.

Subtracting of CMY colorant (because of the interdiction of carbon black colorant) depends on the material being coloured and on qualities of the colorant itself. Many experiments with the determination of CMY for the given $\mathrm{K}$ show finally the regression equation.

The conventional school theory level of relationships: RGB, CMY, CMYK (GCR) is:

$$
\begin{aligned}
& \left(\begin{array}{c}
Y_{0} \\
M_{0} \\
C_{0}
\end{array}\right)=\left(\begin{array}{l}
1 \\
1 \\
1
\end{array}\right)-\left(\begin{array}{l}
B \\
G \\
R
\end{array}\right), \\
& \left(\begin{array}{c}
Y_{k} \\
M_{k} \\
C_{k}
\end{array}\right)=\left(\begin{array}{c}
Y_{0} \\
M_{0} \\
C_{0}
\end{array}\right)-\left(\begin{array}{c}
K \\
K \\
K
\end{array}\right) .
\end{aligned}
$$

This model is not applicable for realistic dyes, colour qualities, material qualities. Colouring with halftones and qualities of material is not included [6].

Our model for determining dependency $X_{30}$ on $X_{0}$ starts with the assumption that each $X_{30}$ colour depends on two other colours. VZ Separation for $K=30 \%$ coverage, is set over the relation:

$X_{30}=\boldsymbol{A} * X_{0}-\boldsymbol{B}$,

where:

$$
\begin{aligned}
X_{0} & =\left(\begin{array}{c}
Y_{0} \\
M_{0} \\
C_{0}
\end{array}\right), \\
\boldsymbol{A} & =\left(\begin{array}{ccc}
-0.02110 & -0.17200 & 1.33200 \\
-0.05620 & 1.31000 & 0.12300 \\
1.12000 & -0.07874 & 0.01650
\end{array}\right), \\
\boldsymbol{B} & =\left(\begin{array}{l}
28.2 \\
30.1 \\
30.2
\end{array}\right) .
\end{aligned}
$$

Matrix $\boldsymbol{A}$ is the result of measuring the twin colour $\mathrm{X}_{0} / \mathrm{X}_{30}$ after printing on polypropylene. Eq. (3) gives colours in the application of $\mathrm{VZ}$ design. Each of three process colours $C, M, Y$ must be greater than $30 \%$ coverage. This is the $V$ area that is characterized by the absence of carbon $K$ components. In the following paper four colours are separated as an example of the invisible, hidden designs.

Assorted colours are twins: purple, dark, grey and greenish. $V$ dyes are given by its three process $\mathrm{C}, \mathrm{M}, \mathrm{Y}$ components, without $K$. However, VZ separation has a default value of $K,(K=30 \%)$ of coverage. $C, M, Y$ are calculated for each dye according to the Eq. (3). For example: $Z_{30}$ dark dye consists of four process components:
$74 \%, 72 \%, 64 \%$ and $30 \% \mathrm{~K}$ give light absorption in the NIR range. Therefore, $K$ is emphasised which means "carbon black" which absorbs $Z$ light well.

Table 1 Four twincolours

\begin{tabular}{|c|c|c|c|}
\hline Colour & $V(\mathrm{CMY}) \%$ & $Z(\mathrm{CMYK}) \%$ & \\
\hline Purple & $40,80,35$ & $11,68,4,30$ & \\
\hline Dark & $90,90,90$ & $74,72,64,30$ & \\
\hline Grey & $45,40,30$ & $24,15,0,30$ & \\
\hline Greenish & $66,33,33$ & $53,3,4,30$ & \\
\hline
\end{tabular}

The coverage of individual colours is given in proportions of three process colours $C, M, Y$ (Tab. 1), according to the Eq. (3). Colour space (Fig. 3 and 4) as V area is determined in the range of 400 to $700 \mathrm{~nm}$. While the human eye sees the light (red component) up to $760 \mathrm{~nm}$, that part $(Z 1)$ of the spectrum is not subjected to the IRD process.

\section{SPECTRAL ANALYSES OF DUAL VZ DYES}

For each experiment of printing twin colours were separately measured. The spectral graph of light absorption is a numerical description of the dyes. The goal of using spectrography was: edit components CMYK process dyes in the IRD procedure reaching the "dual dyes". Spectroscopy helps; in a few steps to reach the good CMYK colour components.

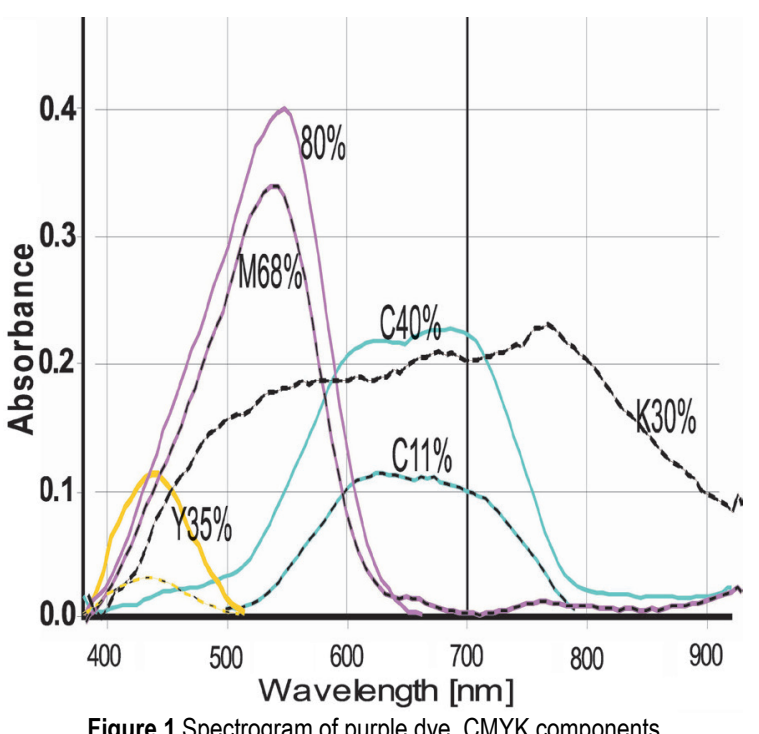

There are tags on the spectrograms that correspond to the values in Tab. 1. The spectrogram indicates the direction of the changes (for next iteration, quality improvement) for each colour $C, M, Y$ colorants. This is an iterative print process of determining the minimum difference of twins in the visual spectrum (Fig. 1). The components of $C, M, Y$ have no absorption light above 760 $\mathrm{nm}$. Yellow colour has a narrow domain; from 400 to 520 $\mathrm{nm}$. Magenta extends up to $650 \mathrm{~nm}$ with peak at $540 \mathrm{~nm}$. Carbon black dyes being given an equal coverage by $30 \%$ have the same value above $900 \mathrm{~nm}$ for all four curved $Z$ 
dyes. The joint purple print is not just a pure addition of spectral values of each component (Fig. 2). The absorption of light depends on the raster shape in print and overlapping each colour component.

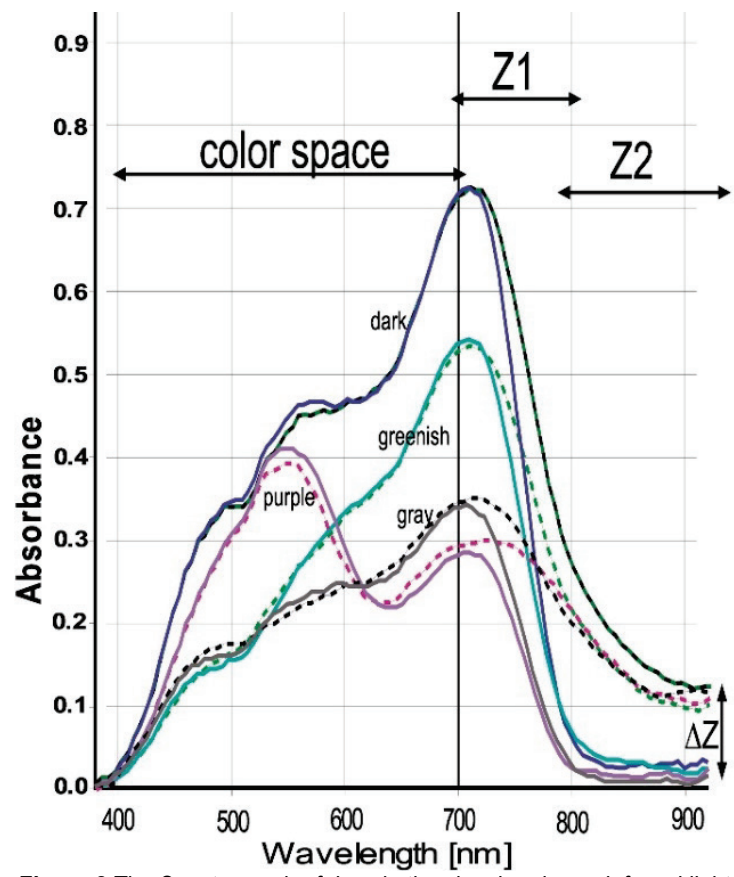

Figure 2 The Spectrograph of dyes in the visual and near infrared light

The spactrograph of dual dyes for the transparent packaging material is divided into three parts: a visual (V) from 400 to $700 \mathrm{~nm}$, a NIR transition (Z1) from 700 to 800 $\mathrm{nm}$ and a hiding area NIR (Z2) from 800 to $1000 \mathrm{~nm}$ [7]. To recognize hidden images, we introduce two parts: $Z 1$ as a transitional area from $V$ to $Z$. In this area, spectrograms of the same twin colours are separated. $Z 2$ is an area in which the absorption of light is measured with $Z$ cameras. The goal is to detect only a $Z$ image by measuring the $\Delta Z$ differences of the visual absorption and $Z$ absorption of twin colours.

The size $\Delta E$ is numerical indicator, degree of twincolours equality in the visual spectrum. The area $Z 1$ is included in the instrumental, quantitative measurement of $\Delta E$. Therefore, the IRD twins can reduce $\Delta E$ below the value of two. The reason is a fixed carbon $K$ component sized by default. The quality evaluation $\Delta E$ is due to the experience of observation of this phenomenon by naked eye.

\section{LINE TRANSPARENT GRAPHICS}

The graphic design consists of three coloured spaces: $V$ colour, $Z$ colour and uncoloured space. Graphic with separated lines enables the visibility, the recognition of the packed product with naked eye. $V$ and $Z$ graphics are built into the constant line, which has the same colour tone, but different components of the process colorants. Constant vector lines are typical design in the area of the security graphics.

The reproduction of line graphs with the silhouette of the butterfly has been prepared for the VZ print process. Fig. 3 is prepared for the four-color process VZ separation (Tab. 1). HIDDEN IMAGES (as butterfly) are designed as a mask, which gives the information of exchange of $V$ or $Z$ colorants with spiral vector lines. The continuous line of the same colour tone changes the amount of process dyes $X_{0} / X_{30}$. The channel $K$ only shows silhouettes of embedded butterflies. This hidden picture is split only in place of coloured lines. Uncoloured empty space denies the data about butterfly.

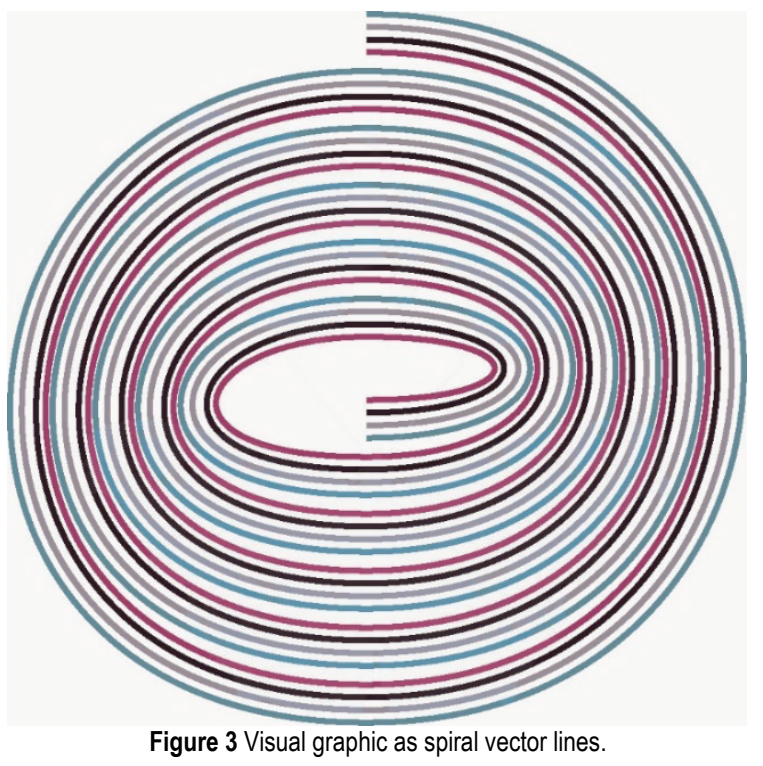

In order to see the packed product, the first image is prepared for the visual range and consists of four spiral flow lines, which are spaced apart. The butterfly image, as the second image, is managed by the VZ separation that will only be extracted with the NIR camera.
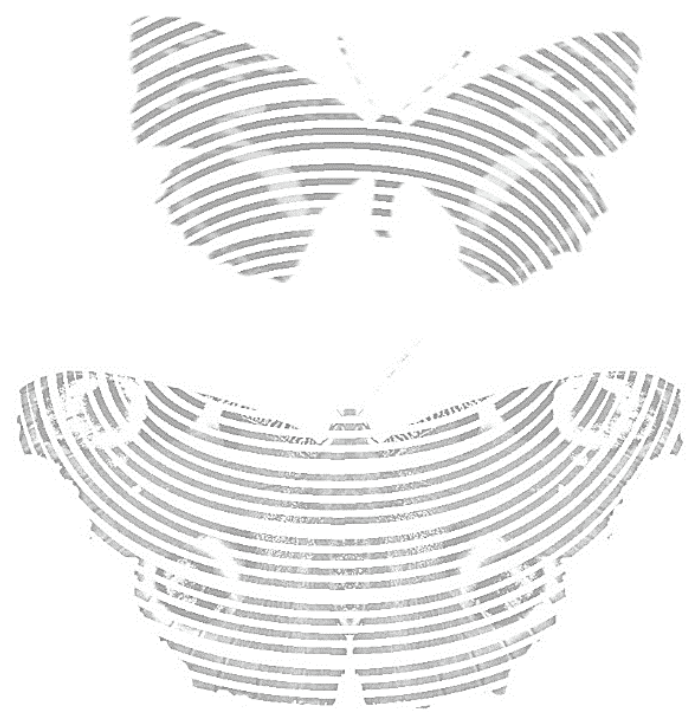

Figure 4 Butterfly infrared silhouette in line graphic

Gradual blocking of light has been shown in the space from 400 to $1000 \mathrm{~nm}$ in order to determine the area of light absorption for $C, M, Y, K$ components. At $720 \mathrm{~nm}$ (stopped display) only Cyan dye remained. Gradual loss or appearance of other process dyes are shown through video animation [8]. The scanning of reproduction is performed with selection of the light wavelengths using the forensic scanner Projectina [9] and is valid for the entire space $V$, and $Z$.

Hidden butterfly is transacted with spiral lines. The butterfly is designed with $Z$ dyes and the environment is 
reproduced with the same $V$ colour tone. Spiral gaps are not covered in the colour of the target because of the transparent design.

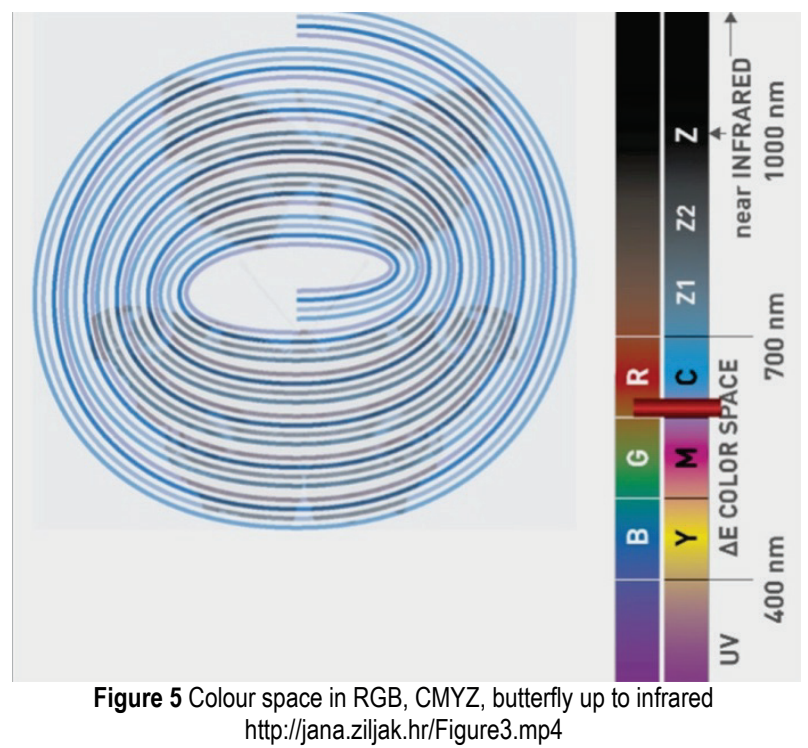

\section{TRANSPARENT GRAPHICS IN FOOD PACKAGING DESIGN}

Spiral graphics has been masked by letter information "1812 GF". The empty space between the spiral lines enables visibility, recognition of the packed product. Extended application is on edible products such as bread, or for example meat. Linear graphic from Fig. 3 is masked on the meat product (ham) with the text coverage of $30 \%$ $K$. IRD transparent labels with hidden text "1812 GF" as invisible $Z$ graphics were added to the dried meat product (Fig. 5 and 6). The VZ separation is subject to the mathematical model with the relations given in Eq. (3). Security graphics for the NIR spectrum is changed in the application. The owner of the product may request that the security graphics have appropriate information for each new release. In the following example, visual graphics (four-color spiral design) remain the same as in Fig. 3 although with different values in the carbon black channel. It will detect and be visible with a $Z$ - infrared camera.

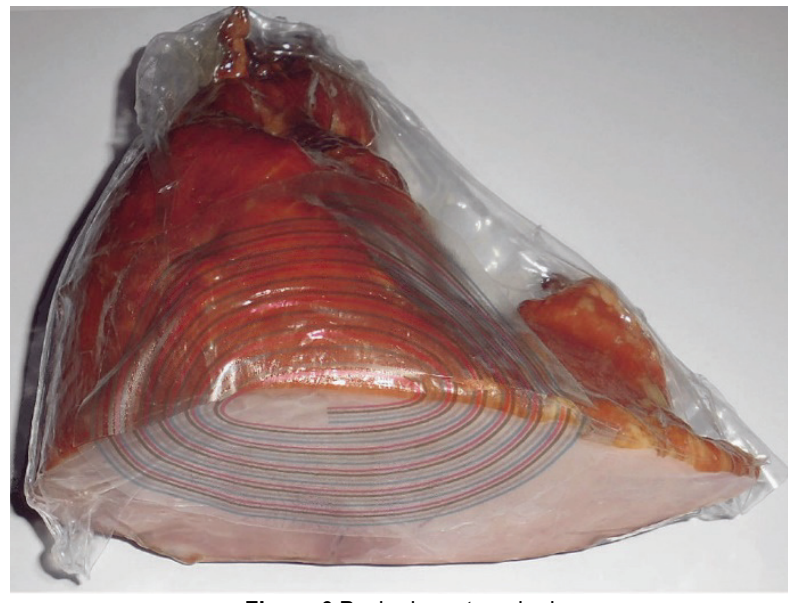

Figure 6 Packed meat marked

Continuous forensic presentation of light blockage from 400 to $1000 \mathrm{~nm}$ is performed as an animation:
http://jana.ziljak.hr/Figure5.mp4 [8]. This is an additional study on process colour domains; their appearance, disappearance, and highlighting of the security information.

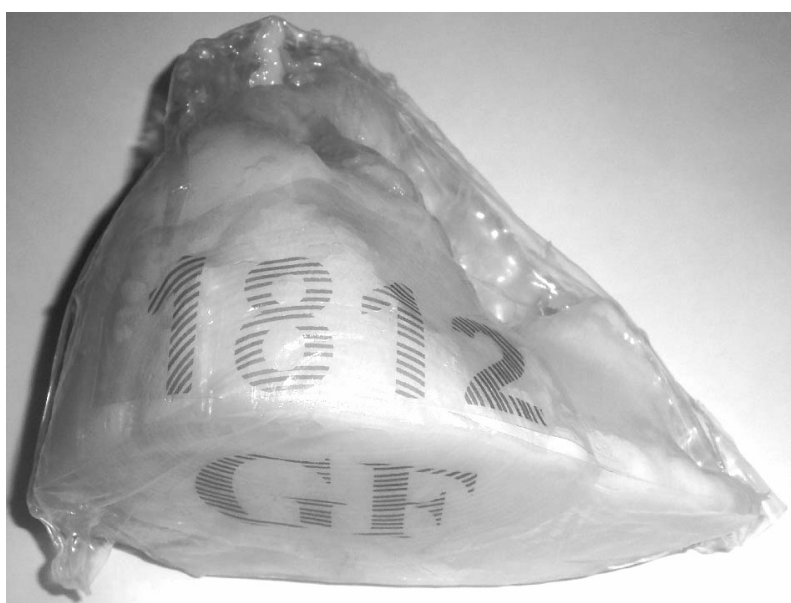

Figure 7 Packed meat with a hidden mark at NIR $1000 \mathrm{~nm}$

\section{CONCLUSION}

Packaging design for a food product is enriched with "infrared printing". This paper provides the technology of double vector line graphics for transparent packaging in the visual and infrared spectrum. The visibility of package products was achieved with spaced lines. The transparent design for packaging is improved with "infrared printing" with VZ prepress graphic procedures.

The general regression mathematical model of the double dyes for VZ separation with process colours for flexographic printing is announced. The hidden image $Z$ is recognizable in the near infrared spectrum. A fixed coverage of $30 \%$ carbon black dyes is determined for such VZ separation as a special form of GCR method. The visual $\mathrm{V}$ dyes, which are only the first part of the $\mathrm{VZ}$ dualcolour collection, do not respond to an infrared camera.

The IRD security graphics prevent counterfeiting packaging without any additional investment, because conventional raw material and printing technologies with process dyes are used. Packaging has double information deriving from visual and "infrared band" dyes. Such graphics cannot be scanned, separated into $V$ and $Z$ state, with the aim of copying the design. The initial visual design is not disturbed. Such technology allows the protection of both the manufacturer of flexible packaging and the manufacturer of the package products. It is not necessary to invest in new technological processes and new raw materials.

\section{REFERENCES}

[1] Pap, K., Žiljak, I., \& Žiljak-Vujic, J. (2010). Image Reproduction for Near Infrared Spectrum and the Infraredesign Theory. Journal of Imaging Science and Technology, 54(1), 10502-1-10502-9(9). https://doi.org/10.2352/J.ImagingSci.Technol.2010.54.1.010502

[2] Žiljak, V., Pap, K., \& Žiljak-Stanimirovic, I. (2011). Development of a prototype for ZRGB infraredesign device. Technical Gazette, 18(2), 153-159. 
[3] Li, C., Wang, C., \& Wang, S. J. (2013). A Black Generation Method for Black Ink Hiding Infrared Security Image. Applied Mechanics and Materials, Trans Tech Publications, Switzerland, 262, 9-12.

[4] Žiljak, J., Tepeš, L., Jurečić, D., \& Žiljak, V. (2017). Hidden infrared graphics on painted canvas. International Journal of Applied Physich, 2, 18-23.

[5] Pogarčić, I., Agić, A., \& Matas, M. (2016). Evaluation of the colorant twins for the neutral grey spectra in infrared graphic procedure. Tehnički vjesnik, 23(6), 1659-1664. https://doi.org/10.17559/TV-20150303132036

[6] Friščić, M., Agić, A., \& Žiljak Stanimirović, I. (2017). Visual and infrared graphic applied through dedicated halftoning for transparent polypropylene packaging. Technical gazette, 24(1), 225-230. https://doi.org/10.17559/TV-20151231105549

[7] Agic, D., Anayath, R., Gršic, J., Agic, A., \& Žiljak, V. (2017). Hidden NIR dual image technology printed on the fabric. Polytechnic \& Design, 5(2), 115-124. https://doi.org/10.19279/TVZ.PD.2017-5-2-05

[8] Web site with animation of the absorption of blocked light in spectral from 400 to $1000 \mathrm{~nm}$, http://jana.ziljak.hr/Figure3.mp4 http://jana.ziljak.hr/Figure5.mp4

[9] Projectina Docucenter 4500, Projectina AG, Switzerland, http://forensictechnology.com/projectina/

\section{Contact information:}

Jana ŽILJAK, dr. sc., associate professor

Zagreb University of Applied Sciences,

Vrbik 8,10000 Zagreb, Croatia

jana@ziljak.hr

jana@tvz.hr

Denis JUREČIĆ, dr. Sc.

University of Zagreb, Faculty of Graphic Arts,

Getaldićeva 2, 10000 Zagreb, Croatia

minerva.graphica.d.0.0@zg.t-com.hr

Vilko ŽlLJAK, dr. sc., emeritus, prof.

University North,

104. brigade 3, 42000 Varaždin, Croatia

vilko@ziljak.hr 